\title{
Resetting of the cardiopulmonary baroreflex 10 years after surgical repair of coarctation of the aorta
}

\author{
D Johnson, H Perrault, S J Vobecky, F Trudeau, E Delvin, A Fournier, A Davignon
}

\begin{abstract}
Objective-To characterise cardiopulmonary baroreflex responses and examine the effects of a 45 minute cycling bout late after successful repair of coarctation of the aorta.

Subjects-10 young adults (mean (SEM) age 18.1 (2.6 years)) operated on for coarctation of the aorta 12.7 (3.5) years earlier, and 10 healthy controls.

Design-Forearm blood flow (venous occlusion plethysmography) and vascular resistance, left ventricular internal diastolic diameter, and central venous pressure estimated from an antecubital vein were measured in the supine position at baseline and during five minute applications of lower

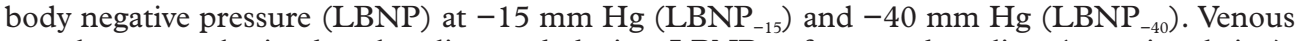
samples were obtained at baseline and during $\mathrm{LBNP}_{-40}$ for noradrenaline (norepinephrine), adrenaline (epinephrine), renin activity, and aldosterone. The tests were repeated after 45 minutes of moderate exercise.

Results-Baseline heart rate (78 (9) $v 64$ (6) beats/min), echocardiographic cardiac output (6.9 (1.1) $v 5.0(0.2) 1 / \mathrm{min}$ ), shortening fraction (41.7 (1.8) \% v $33.3(1.3) \%$ ), and forearm blood flow (3.4 (0.4) $v 2.3(0.3) \mathrm{ml} / 100 \mathrm{~g} / \mathrm{min})$ were higher in the coarctation group than in the controls $(\mathrm{p}<0.05)$. Changes in forearm blood flow and forearm vascular resistance from baseline to $\mathrm{LBNP}_{-40}$ were similar in both groups, but the relation between forearm vascular resistance and estimated central venous pressure or left ventricular internal diastolic diameter was shifted downward in the coarctation group. Plasma adrenaline was increased in the coarctation group (baseline: $3.2(0.6)$ v $2.4(0.3) \mathrm{pmol} / 1$ in controls; $\mathrm{LBNP}_{-40}$ : 687 (151) v 332 (42) pmol/l) (p < 0.05). Both groups showed a similar downward displacement of forearm vascular resistance $(\mathrm{p}<0.05)$ after exercise.

Conclusions-There appears to be resetting of the cardiopulmonary baroreflex to a lower forearm vascular resistance in young adults operated on for coarctation of the aorta, associated with hyperdynamic left ventricular function. Raised circulating adrenaline could contribute to the lower forearm vascular resistance.

(Heart 2001;85:318-325)
\end{abstract}

Physical Education Department, McGill University, 475 Pine Avenue West, Montreal, Quebec, Canada H2W 1S4

H Perrault

Cardiology Unit, Ste-Justine Hospital, Montreal, Quebec, Canada

D Johnson

A Fournier

A Davignon

Cardio-Thoracic

Surgery Unit,

Ste-Justine Hospital

S J Vobecky

Biochemistry

Department,

Ste-Justine Hospital

E Delvin

Physical Activity Sciences Department, UQTR, Quebec,

Canada

F Trudeau

Correspondence to: Dr Perrault

hperra@po-box.mcgill.ca

Accepted 13 November 2000

Keywords: coarctation of aorta; cardiopulmonary baroreflex; forearm vascular resistance; circulating catecholamines

Despite apparently successful surgical repair of coarctation of the aorta in early childhood and the absence of evidence of recoarctation, $10-30 \%$ of patients examined are found to be hypertensive. A notable increase in the prevalence of systolic hypertension is further observed for post-surgical periods greater than 10 years. ${ }^{12}$ Using 24 hour ambulatory blood pressure monitoring, we have recently shown that daytime systolic hypertension was present in $49 \%$ of recordings from patients successfully operated on more than 10 years previously for coarctation of the aorta, compared with $20 \%$ of recordings in patients with a shorter postoperative period. ${ }^{3}$ In the absence of recoarctation or other complication, a clear explanation for this higher incidence of hypertension after repair of coarctation is still lacking.

An attenuated baroreflex has been reported in patients with essential hypertension ${ }^{4-6}$ and it has been proposed that this may be a contributing factor in the evolution of chronic arterial hypertension. There are few data on baroreceptor function after surgical repair of coarctation of the aorta. Alterations in baroreflex function have been described using animal models of experimentally induced coarctation..$^{7-9}$ In rats, Michellini and colleagues reported a diminished arterial baroreflex 10 days after experimental coarctation. ${ }^{9}$ In Labrador puppies, Bonchek and associates reported resetting of the arterial baroreflex to a higher pressure level but normal reflex sensitivity up to two years after experimental coarctation of the aorta. ${ }^{7}$ A similar resetting was also reported in dogs by Igler and colleagues, ${ }^{8}$ associated with an attenuation of baroreflex sensitivity.

There is to our knowledge only one published report of an investigation of arterial baroreflexes following surgical repair of coarctation of the aorta in humans. During routine catheterisation seven years after successful surgical repair, Beekman and colleagues produced increases and decreases in mean arterial pressure of between $10 \%$ and $25 \%$ in six adolescents with mild residual hypertension, using bolus injections of increasing doses of phenylephrine and nitroprusside. ${ }^{10}$ Results showed decreased baroreflex sensitivity as well as resetting about a higher baseline mean arterial pressure. 
In addition to arterial reflexes, inhibitory cardiopulmonary baroreflexes originating mainly from the left atrial and ventricular structures $^{11-13}$ are known to play a role in the regulation of arterial blood pressure. Reflex vascular resistance adjustments to cardiopulmonary baroreceptor deactivation may be investigated in humans through a non-invasive approach using the application of lower body negative pressure and peripheral blood flow measurements.

In this study we used lower body negative pressure at low and high levels to investigate cardiopulmonary arterial baroreflex function in 10 adolescents successfully operated on for coarctation of the aorta at least 10 years before, and in healthy control subjects of similar age. Circulating plasma catecholamines, plasma renin activity, and aldosterone were also measured, as changes in these markers may reflect associated sympathetic or angiotensin II modulating effects. Because an acute bout of moderate dynamic exercise has been seen to induce changes in arterial blood pressure and baroreflex function, ${ }^{14}{ }^{15}$ we repeated measurements following a single 45 minute bout of cycling exercise in patients and controls to compare the pre-exercise and postexercise responses.

\section{Methods}

SUBJECTS

Patients were recruited from a cohort of children operated on for coarctation of the aorta and followed regularly in our paediatric cardiology unit. We investigated 10 normotensive patients operated on at least 10 years earlier and with no evidence of recoarctation (arm-leg pressure gradient less than $15 \mathrm{~mm} \mathrm{Hg}$ ). Surgery had been done at a mean (SEM) age of 5.6 (2.1) years with a mean postsurgical delay of 12.7 (3.5) years. End to end anastomosis had been used in nine patients and left subclavian flap angioplasty in one. Calculation of blood pressure loads as previously reported $^{3}$ showed that patients had a similar daytime prevalence of systolic and diastolic hypertension (14 (5) \% and $1.5(1) \%)$ as controls (12 (4)\% and $1(0.5) \%)$. Electrocardiographic and echocardiographic variables were normal in all patients.

For comparison purposes, a group of 10 healthy adolescents recruited on a voluntary basis from local schools and friends of patients was also evaluated and screened for any known health problems.

The project was approved by the ethics review board of our institution, and informed consent was obtained from all participants, and from the parents or guardian of subjects under 18.

\section{PROCEDURES}

Subjects reported to the laboratory (which was maintained between $19-23^{\circ} \mathrm{C}$ ) in the afternoon for a two hour period. Upon arrival, and at least 30 minutes before starting the protocol, a peripheral venous cannula was inserted into the right antecubital vein for blood sampling and estimation of central venous pressure, with subjects in a supine position. Blood pressure measurements and ECG monitoring in lead III were done throughout the entire protocol.

Forearm blood flow was measured by venous occlusion plethysmography using a mercury in Silastic strain gauge (EC-4, DE Hokanson Inc, Place Bellevue, Washington, USA), as previously described. ${ }^{16}$ The strain gauge was placed 4-5 cm below the antecubital crease on the opposite arm to that used for blood pressure measurements, and the forearm was raised $10 \mathrm{~cm}$ above the anterior chest wall. Venous occlusion pressure was $40-50 \mathrm{~mm} \mathrm{Hg}$ while the circulation to the hand was arrested by inflating a cuff around the wrist at suprasystolic pressure during determination of forearm blood flow. Blood flow measurements were obtained during a two minute hand occluded period, and the mean of at least five blood flow measurements was used for subsequent computations. Forearm vascular resistance was calculated by dividing mean arterial pressure $(\mathrm{mm} \mathrm{Hg})$ by forearm blood flow ( $\mathrm{ml} / \mathrm{min} /$ $100 \mathrm{ml}$ of forearm volume) and is expressed as resistance units.

INDICES OF CENTRAL BLOOD VOLUME

Echocardiographic "sweeps" (HP3500 Sonocardiograph, Hewlett-Packard Inc, Andover, Massachusetts, USA) were made in all subjects, rotating the transducer parallel to the long axis of the left ventricle from the chordae tendinae to the aortic root. Measurements of left ventricular internal diastolic diameter were determined in $\mathrm{M}$ mode after locating the parasternal long axis in cross sectional mode over three consecutive cardiac cycles when heart rate was stable. The end diastolic frame was chosen at the peak of the $R$ wave of the simultaneously recorded ECG. Left ventricular end systolic and end diastolic volumes were derived from the dimensions using the formula of Teichholz and colleagues. ${ }^{17}$ Central venous pressure was also estimated ${ }^{15-20}$ by measuring right venous pressure from an antecubital venous catheter connected through a saline filled line to a pressure transducer (P 23 ID, Gould Instrument Systems, Valley View, Ohio, USA) positioned at the mid-chest level while the arm hung down on the side of the bed in the left lateral supine position. Measurements were obtained at baseline as well as during both levels of lower body negative pressure.

STUDY PROTOCOL AND UNLOADING MANOEUVRES After a 30 minute quiet rest and calibration adjustments, baseline values of blood pressure, heart rate, forearm blood flow, central venous pressure, and circulating hormone concentrations were obtained before application of lower body negative pressure. Baroreflex control of forearm vascular resistance was examined during lower body negative pressure, which deactivates cardiopulmonary receptors and induces reflex vasoconstriction in the forearm, as described previously in detail. ${ }^{21}{ }^{22}$ The subject lay quietly with the lower limbs and body enclosed in an acrylic box sealed by a neoprene skirt at the level of anterosuperior iliac crest. The box was connected to an 
Table 1 Baseline haemodynamic variables in patients and control subjects

\begin{tabular}{lll}
\hline & $\begin{array}{l}\text { Rapaired } \\
\text { coarctation } \\
(n=10)\end{array}$ & $\begin{array}{l}\text { Controls } \\
(n=10)\end{array}$ \\
\hline Heariable & $78(9)^{\star}$ & $64(6)$ \\
SBP (mm Hg) & $117(3)$ & $118(3)$ \\
DBP (mm Hg) & $64(3)$ & $67(3)$ \\
MAP (mm Hg) & $82(2)$ & $84(3)$ \\
eCVP (mm Hg) & $7.1(0.6)$ & $7.7(0.7)$ \\
LVIDD (mm) & $49.0(1.8)$ & $48.9(1.3)$ \\
LVISD (mm) & $28.7(1.9)^{\star}$ & $32.7(1.3)$ \\
Shortening fraction (\%) & $41.7(1.8) \dagger$ & $33.3(1.3)$ \\
Qc (1/min) & $6.9(1.1)$ & $5.0(0.2)$ \\
FBF (ml/100 g/min) & $3.4(0.4)^{\star}$ & $2.3(0.3)$ \\
FVR (units) & $27.0(3.2)^{\star}$ & $43.4(6.3)$ \\
\hline
\end{tabular}

Values are mean $(\mathrm{SEM})$

${ }^{\star} \mathrm{p}<0.05,+\mathrm{p}<0.01 v$ control.

DBP, diastolic blood pressure; eCVP, estimated central venous pressure; FBF, forearm blood flow; FVR, forearm vascular resistance; LVIDD, left ventricular internal diastolic diameter; LVISD, left ventricular internal systolic diameter; MAP, mean arterial pressure; Qc, estimated cardiac output; SBP, systolic blood pressure.

adjustable vacuum source and the pressure within was monitored and recorded throughout the procedure (Gould P 23 ID). Two levels of low body negative negative pressure (LBNP) were applied: $-15 \mathrm{~mm} \mathrm{Hg}\left(\mathrm{LBNP}_{-15}\right)$ and $-40 \mathrm{~mm} \mathrm{Hg}\left(\mathrm{LBNP}_{-40}\right)$, each level being maintained for five minutes. Values of forearm blood flow were taken as the average of five flow curves during the last two minutes of each condition.

During a second series of investigations, lower body negative pressure was applied in an identical fashion for echocardiographic deter-
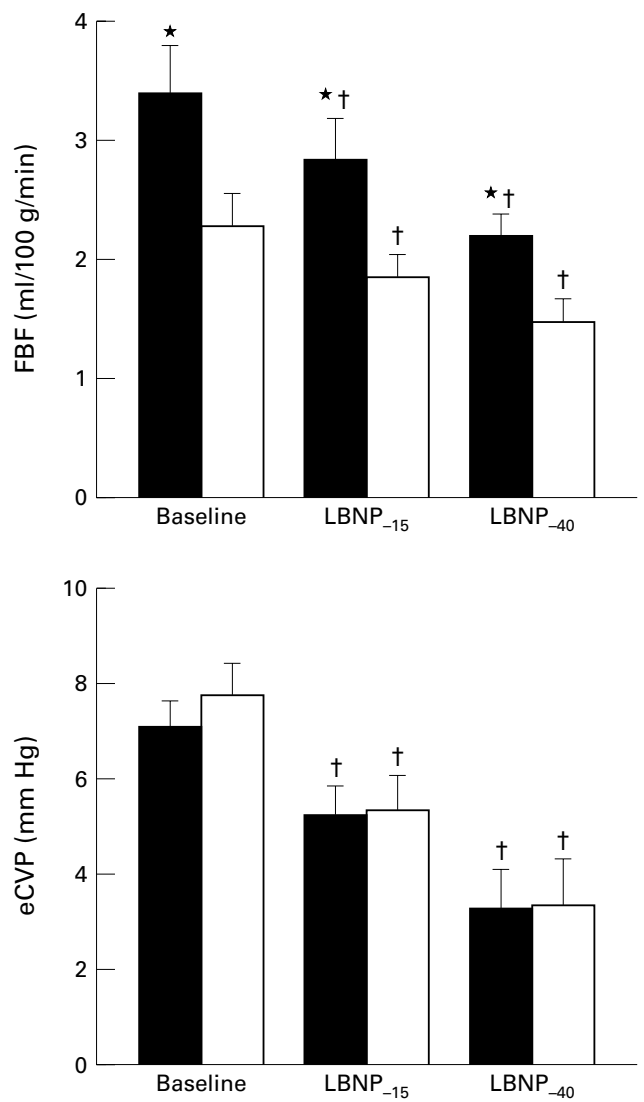

mination of end systolic dimensions and left ventricular internal diameter in diastole, with constant monitoring of heart rate and blood pressure. The order in which we carried out these two series of investigations (forearm blood flow and echocardiography) was randomised.

Forearm blood flow, forearm vascular resistance, and echocardiographic variables were determined at each of the three study end points (baseline, $\mathrm{LBNP}_{-15}$, and $\mathrm{LBNP}_{-40}$ ). Baroreflex control of forearm vascular resistance was examined from the slope of the relation between changes in forearm vascular resistance from baseline and left ventricular internal diastolic diameter and estimated central venous pressure, calculated for each subject and averaged for each group. The same measurements were repeated 30 minutes after termination of a moderate bout of cycling exercise.

\section{EXERCISE}

Exercise was carried out on a cycle ergometer at a submaximal intensity of $70 \%$ of the age predicted maximum heart rate. After a two minute warm up, pedalling resistance was increased until the target heart rate was reached and this exercise load was maintained for a 45 minute period. The exercising workload did not differ between groups, and the subjects had similar heart rates during
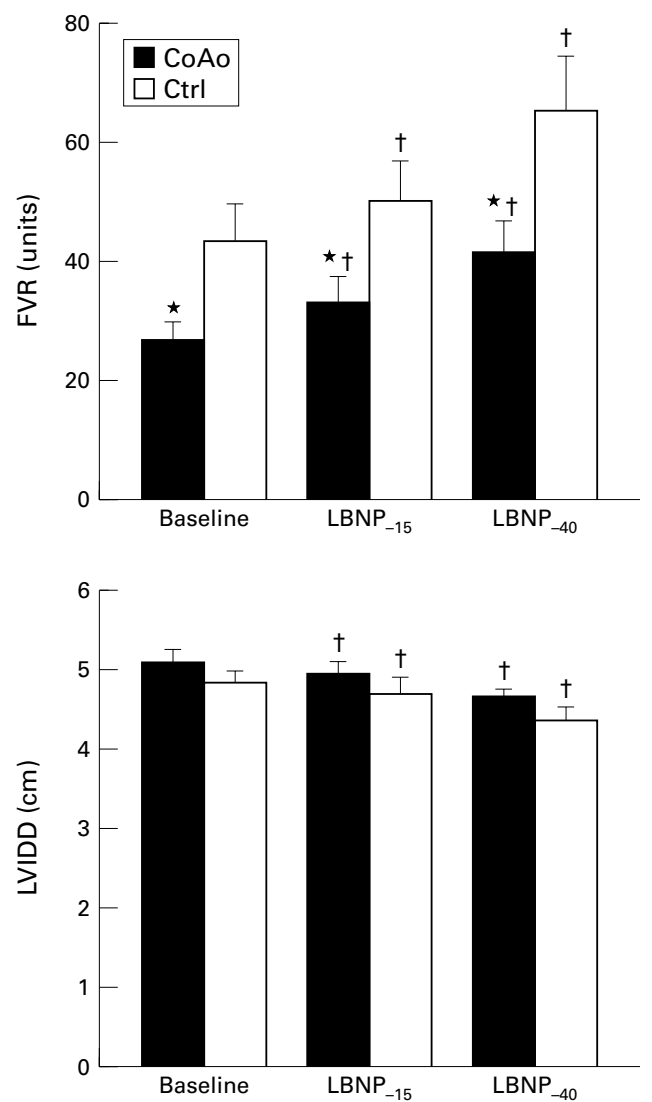

Figure 1 Effects of lower body negative pressure (LBNP) on central vascular volume and forearm responses. Values are means of estimated central venous pressure (eCVP), left ventricular internal diastolic diameter (LVIDD), forearm blood flow $(F B F)$, and forearm vascular resistance (FVR) in patients with coarctation of the aorta (CoAo) and control subjects $(C t r l)$. Error bars $=S E M .{ }^{\star} p<0.05 v$ control subjects; $t p<0.01 v$ baseline. 


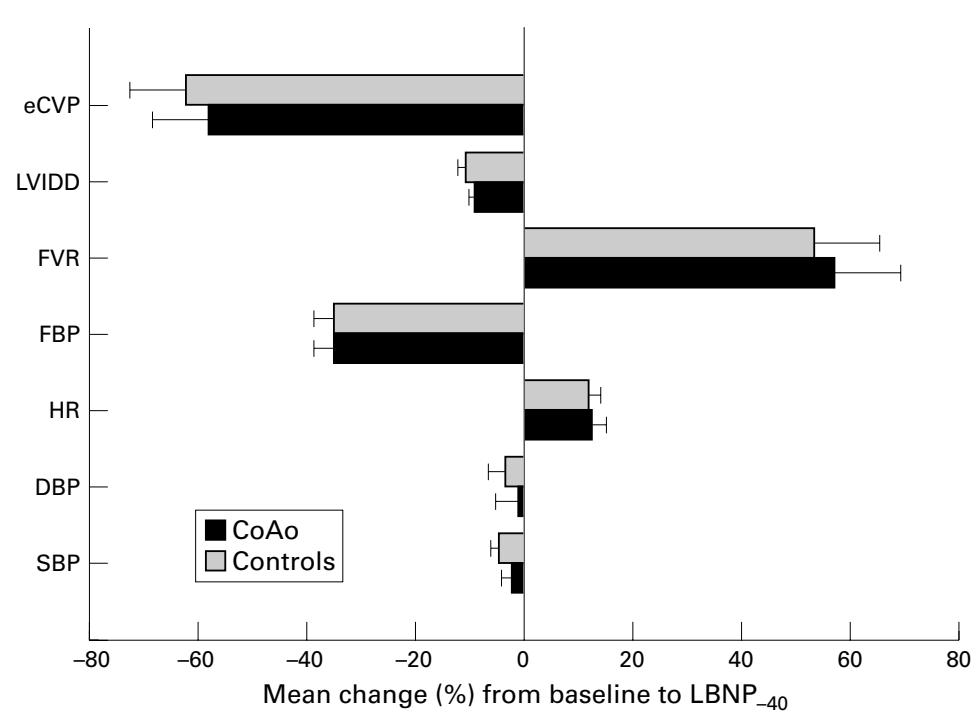

Figure 2 Mean relative changes from baseline at lower body negative pressure (LBNP) of $-40 \mathrm{~mm} \mathrm{Hg}$. Values are means expressed as per cent from baseline for systolic and diastolic blood pressure (SBP, DBP), heart rate (HR), forearm blood flow (FBF), forearm vascular resistance (FVR), left ventricular internal diastolic diameter (LVIDD), and estimated central venous pressure (eCVP) in patients with coarctation of the aorta (CoAo) and controls. Error bars $=$ SEM exercise and similar blood pressures immediately after the exercise bout.

CIRCULATING HORMONAL RESPONSES

Blood samples were withdrawn within 30 seconds after completion of blood flow measurement under baseline conditions, as well as during the last minute of application of $\mathrm{LBNP}_{-40}$. Plasma noradrenaline (norepinephrine) and adrenaline (epinephrine) concentrations were determined using a radioenzymatic method. ${ }^{21}$ Renin activity (ng/l/s) was measured indirectly on EDTA plasma through the generation of angiotensin- 1 and its quantitation by a radioimmunoassay, using ${ }^{125} \mathrm{I}-$ angiotensin-1 as the marker (Incstar Corporation, Stillwater, Minnesota, USA). Aldosterone (pmol/l) was measured by a solid phase radioimmunoassay (Diagnostic Product Corporation, Los Angeles, California, USA), using ${ }^{125} \mathrm{I}-$ aldosterone as the marker. The intra-assay and interassay coefficients of variation for all assays ranged between $7 \%$ and $12 \%$.

\section{STATISTICAL ANALYSES}

All dependent variables are expressed as mean $(\mathrm{SEM})$. A two way $(2 \times 3)$ analysis of variance for repeated measures on the last factor was used to compare the relation between the levels of lower body negative pressure and dependant variables. On finding significant main group or lower body negative pressure effects, the LSD multiple range test was used for further post-hoc comparisons. The slopes of the forearm vascular resistance versus left ventricular internal diastolic diameter and versus estimated central venous pressure relations were calculated individually for each subject by the method of least squares regression. Statistical analyses were performed using Sigma Stat software. Significance was set at $\mathrm{p}<0.05$.

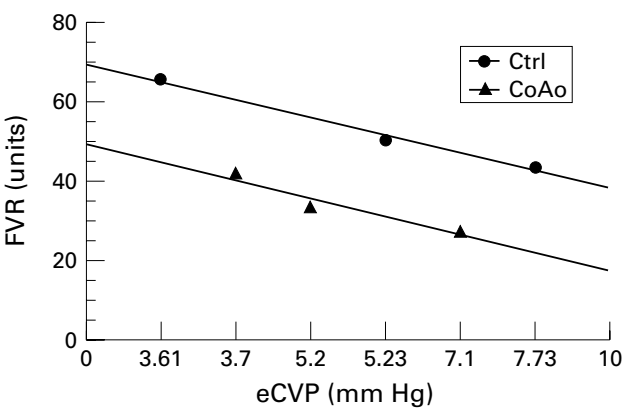

Figure 3 Linear relations between forearm vascular resistance and estimated central venous pressure in coarctation of the aorta and in control subjects. Data points were obtained by averaging individual data during lower body negative pressure at $-40 \mathrm{~mm} \mathrm{Hg}$ and $-15 \mathrm{~mm} \mathrm{Hg}$ and at baseline. Regression lines reflect the mean of the slopes calculated individually for each subject. FVR,

forearm vascular resistance; eCVP, central venous pressure estimated from antecubital venous pressure.

\section{Results}

SUBJECT CHARACTERISTICS

Patients operated on for coarctation of the aorta and control subjects were of similar age (18.1 (2.6) v 21.1 (3.5) years), body weight (63 (4) $v 68$ (3.0) kg), height $(1.7(0.03) v 1.7$ $(0.03) \mathrm{m})$, and body surface area $(1.8(0.01) v$ $\left.1.8(0.1) \mathrm{m}^{2}\right)$.

Baseline haemodynamic data are summarised in table 1 for the coarctation group and the controls. Heart rate was significantly higher in the patients. A similar left ventricular internal diastolic diameter was found in both groups, but the patients had a lower left ventricular internal systolic diameter. Cardiac output was higher in the patients, as was the calculated left ventricular shortening fraction. Systolic, diastolic, and mean arterial pressure and estimated central venous pressure did not differ between the groups. However, forearm blood flow was higher and forearm vascular resistance lower in the coarctation group than in the controls.

\section{VASOMOTOR AND HEART RATE RESPONSES TO} BARORECEPTOR UNLOADING

Figure 1 shows the effects of lower body negative pressure on indices of central volumenamely, estimated central venous pressure and left ventricular internal diastolic diametertogether with forearm blood flow and forearm vascular resistance. As expected, in both groups application of negative pressure decreased the estimated central venous pressure and left ventricular internal diastolic diameter $(\mathrm{p}<0.05)$, thus confirming an unloading effect on central blood volume. $\mathrm{LBNP}_{-15}$ and $\mathrm{LBNP}_{-40}$ caused significant stepwise reductions in forearm blood flow and increases in forearm vascular resistance in both groups. Despite a similar pattern of vasoconstrictor response, patients showed a greater forearm blood flow, and inversely a lower forearm vascular resistance, under both levels of lower body negative pressure.

The mean relative changes from baseline induced by $\mathrm{LBNP}_{-40}$ application are shown in fig 2. Application of lower body negative pressure resulted in only slight changes of systolic and diastolic pressures. In keeping with a 

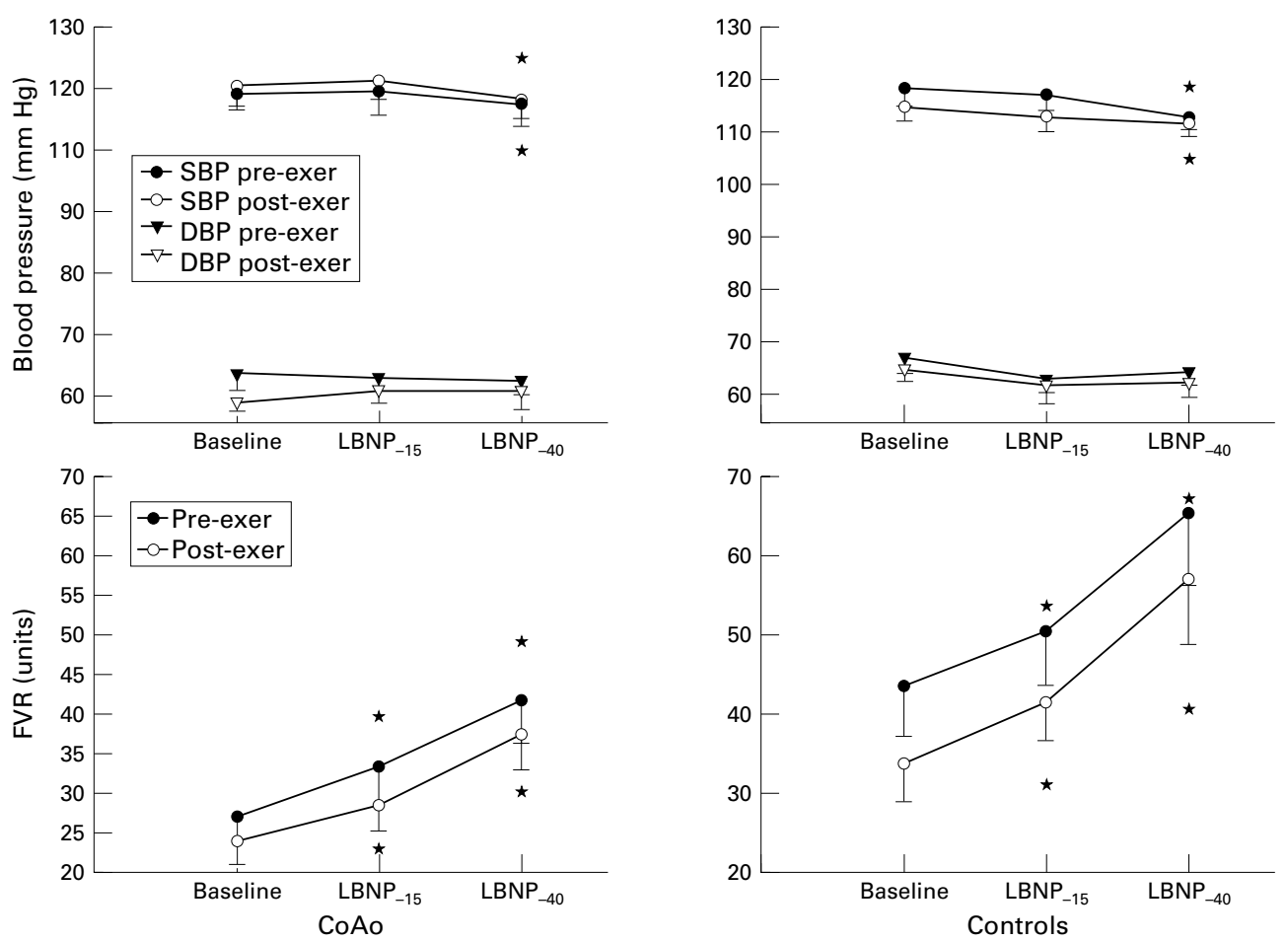

Figure 4 Effects of a single 45 minute cycling bout on systolic and diastolic blood pressure (SBP, DBP) as well as on forearm vascular resistance (FVR) responses to lower body negative pressure (LBNP). Results in patients with coarctation of the aorta $(\mathrm{CoAo})$ are on the left and those of controls are on the right. Values are means, error bars $=S E M .{ }^{\star} p<0.05 v$ baseline.

stimulation of the arterial baroreflex at higher levels of lower body negative pressure, a $12 \%$ increase in heart rate was observed in response to $\mathrm{LBNP}_{-40}$, the magnitude of the response being similar in the two groups. The extent of the decrease in forearm blood flow and the increase in forearm vascular resistance induced by lower body negative pressure was similar in the two groups, as were changes in left ventricular internal diastolic diameter and estimated central venous pressure.

The sensitivity of the baroreflex function, illustrated by the relation between the estimated central venous pressure and the corresponding calculated forearm vascular resistance, is shown in fig 3, using observations at baseline and at $-15 \mathrm{~mm} \mathrm{Hg}$ and $-40 \mathrm{~mm} \mathrm{Hg}$ lower body negative pressure. Parallel relation lines were found for patients and controls,

Table 2 Adrenergic and renin-angiotensin-aldosterone system responses to lower body negative pressure at $-40 \mathrm{~mm} \mathrm{Hg}\left(\mathrm{LBNP}_{-40}\right)$ in patients with repaired coarctation and control subjects

\begin{tabular}{|c|c|c|c|}
\hline Hormone & Baseline & $L B N P_{-40}$ & Change (\%) \\
\hline \multicolumn{4}{|c|}{ Noradrenaline $(\mathrm{nmol} / \mathrm{l})$} \\
\hline Coarctation & $3.2(0.6)$ & $5.5(0.5) \ddagger^{\star}$ & $90.6(23.5)$ \\
\hline Controls & $2.4(0.3)$ & $3.7(0.3)^{\star \star}$ & $57.3(15.5)$ \\
\hline \multicolumn{4}{|c|}{ Adrenaline (pmol/1) } \\
\hline Coarctation & $406.7(94.9) \dagger$ & $688.6(151.2) \ddagger$ & $87.5(15.0)$ \\
\hline Controls & $231.9(42.6)$ & $331.7(41.7)$ & $71.9(29.3)$ \\
\hline \multicolumn{4}{|c|}{ Plasma renin activity (ng/1/s) } \\
\hline Coarctation & $0.37(0.09)$ & $0.49(0.17)$ & $57.7(62.6)$ \\
\hline Controls & $0.27(0.07)$ & $0.36(0.16)$ & $32.8(32.0)$ \\
\hline \multicolumn{4}{|c|}{ Aldosterone (pmol/1) } \\
\hline Coarctation & $217.9(38.2)$ & $186.3(49.9)$ & $-18.2(9.6)$ \\
\hline Controls & $235.1(50.0)$ & $180.6(55.4)$ & $-17.2(9.2)$ \\
\hline
\end{tabular}

Values are mean (SEM)

$t \mathrm{p}<0.05$ between groups; $\neq \mathrm{p}<0.01$ between groups; ${ }^{\star} \mathrm{p}<0.05 v$ baseline; ${ }^{\star \star} \mathrm{p}<0.01 v$ baseline. although a downward shift in the forearm vascular resistance line is seen in the coarctation group. A similar pattern was also found when using left ventricular internal diastolic diameter as central volume index (not shown). The mean slope calculated from slopes of each individual estimated central venous pressureforearm vascular resistance regression line was not significantly different between groups $(-5.48(0.82) v-7.87(1.53))$.

EFFECTS OF EXERCISE

As can be seen from fig 4, systolic and diastolic blood pressures were not significantly changed under any testing condition. However, exercise resulted in a significant downward displacement of the forearm vascular resistance and an upward shift of forearm blood flow (not shown) in both patients and controls.

HORMONAL RESPONSES TO BARORECEPTOR UNLOADING

The circulating catecholamine and reninaldosterone hormonal profiles assessed at baseline and at $\mathrm{LBNP}_{-40}$ are shown in table 2 . At baseline, patients showed slightly but non-significantly higher noradrenaline concentrations and plasma renin activity, and significantly higher concentrations of adrenaline. A significant increase in plasma noradrenaline from baseline was seen in both groups in response to $\mathrm{LBNP}_{-40}$. As can be seen from calculated changes relative to baseline, $\mathrm{LBNP}_{-40}$ increased circulating catecholamines and plasma renin activity but decreased circulating aldosterone concentrations to the same extent in both patients and controls. 


\section{Discussion}

Although we were only able to study a small number of subjects, our results suggest that normotensive adolescents operated on for coarctation of the aorta in early childhood have a downward shift in the regression line between forearm vascular resistance and estimated central venous pressure or left ventricular internal diastolic diameter, suggesting a resetting of the integrated cardiopulmonary-arterial baroreflex without change in its sensitivity. This was not associated with differences in sympathetic or renin-aldosterone hormone status or responsiveness to lower body negative pressure or exercise.

BAROREFLEX DISTURBANCES IN ESSENTIAL HYPERTENSION AND OPERATED COARCTATION OF THE AORTA

Recent observations obtained in our laboratory using 24 hour blood pressure monitoring indicate that despite successful surgical repair of coarctation of the aorta in early childhood, asymptomatic young adult patients have up to twice the hypertension load of their age matched control counterparts 10 years or more after surgery. ${ }^{3}$ Contrary to the relatively large body of evidence showing a resetting of baroreceptor cardiac reflexes in subjects with borderline or pronounced hypertension, ${ }^{62-26}$ there have been few investigations of arterial reflex involvement in patients operated on for coarctation of the aorta, and to our knowledge there are no data on the cardiopulmonary baroreflex.

Igler and colleagues showed a return towards normal carotid sinus baroreceptor function 3-7 months after surgical repair of coarctation. ${ }^{8}$ A resetting of the arterial baroreflex to operate at higher arterial pressures in response to injections of pressor and depressor drugs was also found in mildly hypertensive adolescents operated on for coarctation seven years earlier. ${ }^{10}$

To our knowledge, our study is the first to examine cardiopulmonary baroreflex function in patients after surgery for coarctation of the aorta. Our results do not support the view that a change in cardiopulmonary baroreflex sensitivity is a factor in the higher prevalence of hypertension seen years after surgical repair of coarctation. An increase in cardiopulmonary baroreflex sensitivity is generally reported in the early stages of hypertension, followed by a decrease in response gain with increasing severity. ${ }^{22} 24-27$ In the present study, estimates of baroreflex sensitivity in patients and controls were similar. Low level suction (10$20 \mathrm{~mm} \mathrm{Hg}$ ) to the lower body decreases central blood volume with little observable effect on heart rate or mean arterial pressure, and its effect is thus considered to be primarily on cardiopulmonary baroreflexes. On the other hand, higher levels of negative pressure which produce a fall in pulse pressure and result in a reflex chronotropic and vasomotor response affect both cardiopulmonary and arterial baroreflexes..$^{13}$ A downward shift in the forearm vascular resistance to estimated central venous pressure relation was seen in the coarctation group, suggesting a resetting to a lower central venous pressure threshold for the release of vasoconstrictor tone. Because the forearm vascular resistance versus central venous pressure relation was obtained over the low and high ranges of negative pressure application, this response reflects the combined cardiopulmonary and arterial reflex influences. We cannot therefore draw any conclusions about specific cardiopulmonary or arterial baroreflex functions from this study.

VASCULAR RESISTANCE AFTER SURGICAL REPAIR OF COARCTATION OF THE AORTA

There are few data on measurements of vascular resistance after repair of coarctation of the aorta. Our present results, obtained using venous occlusion plethysmography, confirm previous observations in our laboratory showing lower femoral, humeral, and renal arterial resistance in patients successfully operated on for coarctation of the aorta and with normal resting blood pressure values. ${ }^{28}$ Differences in left arm vascular development have been described following correction of coarctation of the aorta using the subclavian flap surgical procedure. ${ }^{29}{ }^{30}$ However, in the present study the end to end anastomosis procedure was used in all but one of the patients. It thus appears unlikely that the lower vascular resistance found results from abnormal vascular development. Functional abnormalities, such as a reduced hyperaemic response to a three minute arm occlusion and a limited vasodilator response to glyceryl trinitrate infusion, have also been reported some $7-17$ years after repair of coarctation of the aorta, ${ }^{31} 32$ which is compatible with a more pronounced baseline vasodilatation.

Previous observations in rats have shown that an increase in the discharge rate of afferent C fibres located in the wall of the left ventricle-caused by a series of positive inotropic interventions - resulted in a greater inhibition of vasoconstriction influences. ${ }^{33}{ }^{34} \mathrm{~A}$ change in the ventricular contractile state could thus account for a resetting of the cardiopulmonary reflex response. Baseline echocardiographic measurements obtained in our patients indicate a hyperdynamic cardiac output related to a higher heart rate and increased left ventricular shortening fraction. This is in agreement with previous observations showing the persistence of enhanced left ventricular systolic function and an overall hyperdynamic circulation both at rest and during exercise ${ }^{35-37}$ long after surgical repair of coarctation of the aorta. In the present study, patients had nearly twice the adrenaline concentration of controls, which could contribute to the exaggerated ventricular contractile function. A chronic hypercontractile ventricular state in coarctation of the aorta could thus enhance cardiopulmonary afferent receptor stimulation, leading to further sympathoinhibitory influences and lower vascular resistance. In addition, the raised circulating adrenaline could, at least in part, contribute to the lower vascular resistance through the enhanced $\beta_{2}$ adrenergic vasodilator response that is known to occur in skeletal muscle vasculature. 
HORMONAL RESPONSES TO LOWER BODY

NEGATIVE PRESSURE APPLICATION

Little information is available on circulating catecholamine and renin-aldosterone responses in the long term follow up of patients operated on for coarctation of the aorta. The present results are in agreement with previous reports $^{38-42}$ showing baseline plasma noradrenaline and aldosterone concentrations, as well as plasma renin activity, to be in the normal range of values for adolescents having successful surgery in early childhood. Raised plasma renin activity has, however, also been reported in the long term follow up of coarctation of the aorta, and was associated with an increase in adrenergic stimulation, presumably resulting from a lower renal perfusion secondary to a potential recoarctation. In the present study, signs of aortic restenosis resulted in exclusion from the experimental protocol.

Our findings nevertheless showed higher circulating adrenaline in our coarctation group than in controls. Raised adrenaline concentrations in coarctation of the aorta have previously been reported in the early postoperative period after surgical repair, ${ }^{39}$ though such data can hardly be compared with ours, obtained some 10 years after surgery. The reason for our observation thus remains unclear.

BAROREFLEX FUNCTION FOLLOWING EXERCISE

Our finding of a significantly lower forearm vascular resistance in both groups following exercise is in line with reductions in forearm vascular resistance previously reported after a single bout of exercise of moderate intensity in patients with mild to moderate hypertension. ${ }^{14}{ }^{15}$ Despite the baseline resetting to lower forearm vascular resistance in the patient group, exercise induced a further downward shift in the estimated central venous pressureforearm vascular resistance regression in both groups (data not shown). It did not alter the slope of the regression line and thus did not affect the sensitivity.

Forearm vascular resistance was decreased to the same extent in patients and controls. This could be related to enhanced vasodilatation secondary to exercise induced thermoregulatory response to the same exercise induced metabolic demand.

CONCLUSIONS

Measurements of forearm blood flow by venous occlusion plethysmography during lower body negative pressure suggest a resetting of the cardiopulmonary baroreflex to a lower level of vascular resistance in normotensive adolescents after successful surgery for coarctation in early childhood. The respective contributions of arterial and cardiopulmonary baroreflexes remain to be determined. Echocardiographic measurements indicate a hyperdynamic contractile state, which could also be related to an observed increase in circulating adrenaline in our coarctation group. In view of the relatively small number of patients in our study group, further investigations are needed to confirm our findings and to examine the functional significance of the lowered peripheral vascular resistance.

This work was supported in part by a doctoral fellowship from the "Formation de Chercheurs et l'Aide à la Recherche" programme of the Quebec Ministry of Education and from the graduate faculty of the University of Montreal to DJ.

1 Zehr KJ, Gillinov M, Redmond JM, et al. Repair of coarctation of the aorta in neonates and infants: a thirty-year tion of the aorta in neonates and infants:

2 Clarkson PM, Nicholson MR, Barrat-Boyes BG, et al. Results after repair of coarctation of the aorta beyond infancy: a 10 to 28 year follow-up with particular reference to late systemic hypertension. Am f Cardiol 1983:51:14818.

3 Johnson D, Perrault H, Vobecky SJ, et al. Influence of the postoperative period and surgical procedure on ambulatory blood pressure-determination of hypertension load after successful surgical repair of coarctation of the aorta. Eur Heart f 1998;19:638-46.

4 Radaelli A, Bernardi L, Valle F, et al. Cardiovascular autonomic modulation in essential hypertension. Effect of tilting. Hypertension 1994;24:556-63.

5 Meyrelles SS, Tinucci T, Hollanda HEM, et al. Baroreflex control of muscle sympathetic nerve activity in mild hypertension. Am f Hypertens 1997;10:162-7.

6 Mancia G, Grassi G, Ferrari AU. Reflex control of the circulation in experimental and human hypertension. In: Zanchetti A, Mancia G, eds. Handbook of hypertension, vol 17: Pathophysiology of hypertension. Amsterdam: Elsevier 17: Pathophysiology of

7 Bonchek LI, Doctor A, Rees DM. Baroreflex sensitivity and carotid sinus dimensions in dogs with coarctation. $\mathcal{F} A p p l$ Physiol 1976;41:36-40.

8 Igler FO, Boerboom LE, Werner PH, et al. Coarctation of the aorta and baroreceptor resetting: a study of carotid baroreceptor stimulus-response characteristics before and after surgical repair in the dog. Circ Res 1981;48:365-71.

9 Michelini LC, de Oliveira M, dos Santos M. Baroreceptor reflex control of heart rate during development of coarctation of the aorta. Hypertension 1992;19(suppl II):II-15963.

10 Beekman RH, Katz BP, Moorehead-Steffens C, et al. Altered baroreceptor function in children with systolic hypertension after coarctation repair. Am f Cardiol hypertension after

11 Hainsworth R. Reflexes from the heart. Physiol Rev 1991;71:617-58

12 Oren RM, Schobel HP, Weiss RM, et al. Importance of left atrial baroreceptors in the cardiopulmonary baroreflex of normal humans. F Appl Physiol 1993;74:2672-80.

13 Wieling W, Wesseling KH. Importance of reflexes in the circulatory adjustments to postural changes. In: Hainsworth $\mathrm{R}$, Makt AL, eds. Cardiovascular reflex control in health and disease. London: WB Saunders, 1993.

14 Cléroux J, Kouame N, Nadeau A, et al. After effects of exercise on regional and systemic hemodynamics in hypertension. Hypertension 1992;19:183-91.

15 Cléroux J, Kouame N, Nadeau A, et al. Baroreflex regulation of forearm vascular resistance after exercise in hypertensive and normotensive humans. Am f Physiol 1992;263:H1523-31.

16 Greenfield A, Whitney RJ, Mowbray JF. Methods for the investigation of peripheral blood flow. Br Med Bull 1963;19:101-9.

17 Teichholz LE, Kreulen T, Herman MV, et al. Problems in echocardiographic volume determinations: echocardiographic-angiographic correlations in the presence or absence of asynergy. Am f Cardiol 1976;37:7-1 1 .

18 Gauer OH, Sieker HO. The continuous recording of central venous pressure changes from an arm vein. Circ Res 1956; 4:74-8.

19 Kouamé N, Nadeau A, Lacourcière Y, et al. Effects of different training intensities on the cardiopulmonary baroreflex control of forearm vascular resistance in hypertensive subjects. Hypertension 1995;25:391-8.

20 Mack GW, Shi X, Nose H, et al. Diminished baroreflex control of forearm vascular resistance in physically fit humans. f Appl Physiol 1987;63:105-10.

21 Giannattasio C, Seravalle G, Bolla GB. Cardiopulmonary receptor reflexes in normotensive athletes with cardiac receptor reflexes in normotensive athlet.

22 Grassi G, Giannattasio C, Cléroux J, et al. Cardiopulmonary reflex before and after regression of left ventricular hypertrophy in essential hypertension. Hypertension 1988; 12:227-37.

23 Peuler JD, Johnson GA. Simultaneous single isotope radioenzymatic assay of plasma norepinephrine, epinephrine and dopamine. Life Sci 1977;21:625-36.

24 Mark AL, Kerber RE. Augmentation of cardiopulmonary baroreflex control of forearm vascular resistance in borderline hypertension. Hypertension 1982;4:39-46.

25 Zanchetti A, Mancia G. Cardiovascular reflexes and hypertension. Hypertension 1991;18(suppl III):III-13-21.

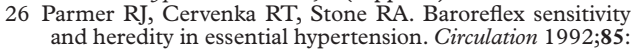
497-503.

27 Head GA. Baroreflexes and cardiovascular regulation in hypertension. I Cardiovasc Pharmacol 1995;26(suppl 2): 
28 Johnson D, Bonnin P, Perrault H, et al. Peripheral blood flow responses to exercise after successful correction of coarcta(1995;26:1719-2

29 Shenberger JS, Prophet SA, Waldhausen JA, et al. Left subclavian flap aortoplasty for coarctation of the aorta: effects on forearm vascular function and growth. $\mathcal{f} \mathrm{Am}$ Coll Cardio 1989;14:953-9.

30 Todd PJ, Dangerfield PH, Hamilton DI, et al. Late effects on the left upper limb of subclavian flap aortoplasty. $\mathcal{F}$ Thorac Cardiovasc Surg 1983;85:678-81.

31 Guenthard J, Wyler F. Exercise-induced hypertension in the arms due to impaired arterial reactivity after successful coarctation resection. Am f Cardiol 1995;75:814-17.

32 Gardiner HM, Celermajer DS, Sorensen KE, et al. Arterial reactivity is significantly impaired in normotensive young adults after successul repair of aortic coarctation in childhood. Circulation 1994;89:1745-50.

33 Thoren P. Atrial receptors with vagal afferents in the cat. Discharge frequency and pattern in relation to atrial Discharge frequency and pattern
pressure. Circ Res 1976;38:357-62.

34 Thoren PN. Characteristics of left ventricular receptors with nonmedullated vagal afferents in cats. Circ Res 1977;40 nonmed

35 Moskowitz WB, Schieken RM, Mosteller M, et al. Altered systolic and diastolic function in children after "successful" repair of coarctation of the aorta. Am Heart $\mathcal{F} 1990 ; 120$ 103-9.
36 Kimball BP Shurvell BL, Houle S, et al. Persistent ventricular adaptations in postoperative coarctation of the aorta. $\mathcal{F}$ Am Coll Cardiol 1986;8:172-8.

37 Carpenter MA, Dammann JF, Watson DD, et al. Left ventricular hyperkinesia at rest and during exercise in normotensive patients 2 to 27 years after coarctation repair. $\mathcal{F}$ Am Coll Cardiol 1985;6:879-86.

38 Sehested J, Kornerup HJ, Pedersen EB, et al. Effects of exercise on plasma renin, aldosterone and catecholamines before and after surgery for aortic coarctation. Eur Heart $\mathcal{F}$ 1983;4:52-8.

39 Benedict CR, Grahame-Smith DG, Fisher A. Changes in plasma catecholamines and dopamine beta-hydroxylase after corrective surgery for coarctation of the aorta. Circulation 1978;57:598-602

40 Parker FB, Streeten DHP, Farrell B, et al. Preoperative and postoperative renin levels in coarctation of the aorta. Circulation 1982;66:513-14.

41 Simsolo R, Grunfeld B, Gimenez M, et al. Long-term systemic hypertension in children after successful repair of coarctation of the aorta. Am Heart f 1988;115:1268-73.

42 Ross RD, Clapp SK, Gunther S, et al. Augmented norepinephrine and renin output in response to maximal exercise in hypertensive coarctectomy patients. Am Heart f 1992;123: $1293-9$

\section{Magnetic resonance image of a large, fatal angiosarcoma of the heart}

A 49 year old man underwent kidney transplantation. Because of an initial rejection of the allograft the usual doses of azathioprine and steroids were increased. Three years later the patient developed atrial fibrillation and clinical features of pericarditis. An echocardiogram showed a right atrial mass which was confirmed by computed tomography. Magnetic resonance imaging with gadolinium injection revealed the true extent of the tumour and indicated a diagnosis of angiosarcoma of the heart (below left, arrows show the tumour invading the interventricular septum, right atrium and right ventricle walls; below right, arrows show the reduction of the right atrium and involve ment of the atrioventricular septum by the expansive mass). Because of major involvement of the cardiac

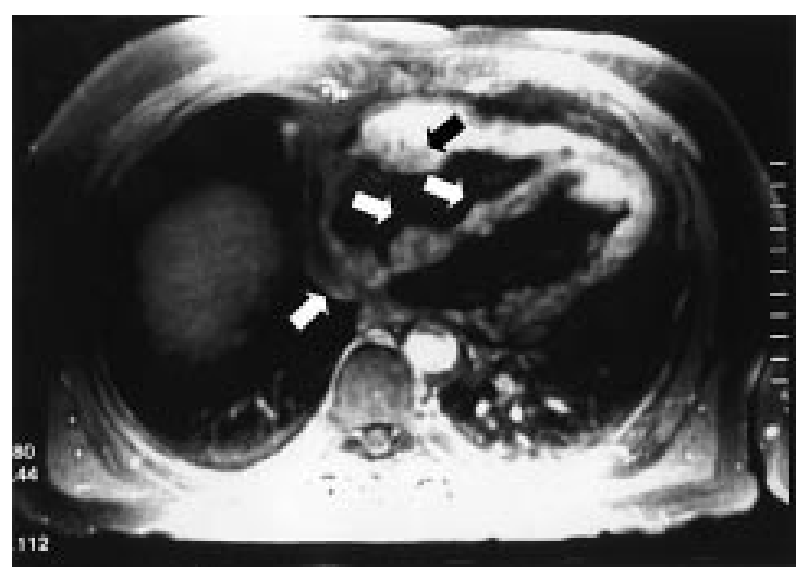

structures it was decided to initiate chemotherapy. However, because of a refractory heart and renal failure, the patient died in the following month. The diagnosis was confirmed by necropsy, histology, and electron microscopy.

Angiosarcoma is a rare tumour of the heart and has a poor prognosis. In our case the extent of the tumour was exceptional and accounts for the rapid death of the patient. Magnetic resonance imaging with gadolinium injection appears to be the imaging modality of choice for an early diagnosis of malignant heart tumours.

GIANLUCA RIGATELLI EMANUELA VISENTIN GIORGIO RIGATELLI

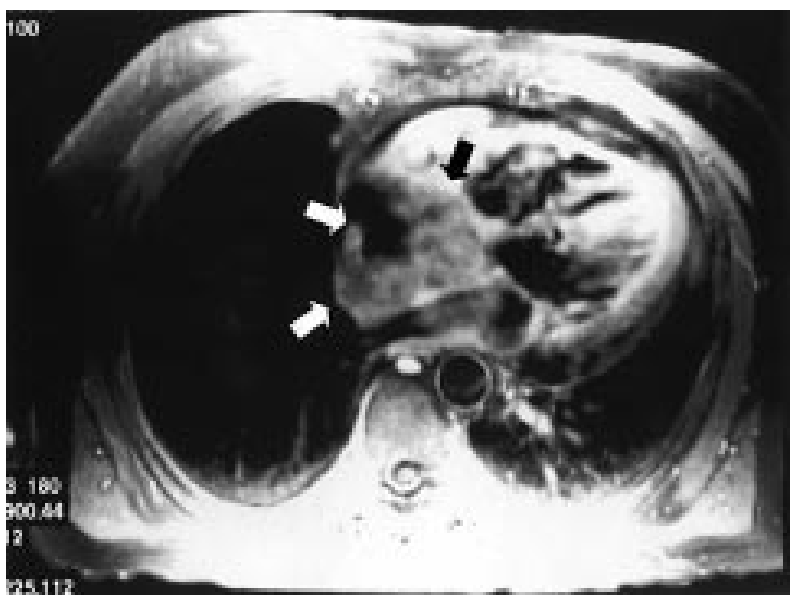

